\title{
A Method for Computing Bessel Function Integrals
}

\author{
By Peter Linz
}

\begin{abstract}
Infinite integrals involving Bessel functions are recast, by means of an Abel transform, in terms of Fourier integrals. As there are many efficient numerical methods for computing Fourier integrals, this leads to a convenient way of approximating Bessel function integrals.
\end{abstract}

1. Introduction. Integrals involving Bessel functions of the form

$$
I(\rho)=\int_{0}^{\infty} f(x) J_{0}(\rho x) d x
$$

occur in many physical applications, especially in the solution of certain mixed boundary value problems (cf. Sneddon [8], [9]). An extensive collection of known integrals of this type may be found in [4]. However, for many functions $f(x)$, no closed form solution is known and it becomes necessary to use numerical techniques. The numerical approach is complicated by the fact that, if $f(x)$ goes to zero slowly as $x \rightarrow \infty$, the integration has to be performed over many oscillations of $J_{0}(x)$, and the standard numerical techniques become inefficient. Furthermore, positive and negative contributions to the integral tend to cancel and, hence, lead to a high loss of significance. The situation is similar to the one encountered in the numerical evaluation of Fourier integrals

$$
C(\omega)=\int_{0}^{\infty} f(x) \cos \omega x d x .
$$

A great many efficient methods have been developed to treat (2). (E.g. [1], [2], [5]; for a summary, see [3, p. 59]. More recent work is described by Lyness in [7].) On the other hand, the integral (1) seems to have received very little attention (for one special method, see [6]). One could, of course, attempt to generalize the methods developed for Fourier integrals to the Bessel function case, but there exists a simpler way. As we will show below, integrals of the form (1) can be expressed in terms of Fourier integrals, so that one can use all the techniques developed for Fourier integrals to evaluate Bessel function integrals. In the subsequent analysis, we will assume that $\lim _{x \rightarrow \infty} f(x)=0$, and that $f(x)$ is such that all formal manipulations are justified.

2. An Application of Abel Transforms. We introduce the Abel transform of $I(\rho)$, defined by

Received June 21, 1971.

AMS 1970 subject classifications. Primary 65D30; Secondary 35A40, 42A68, 44A20.

Key words and phrases. Bessel functions, Fourier-Bessel coefficients, Hankel transforms, numerical integration.

Copyright $\odot$ 1972, American Mathematical Society 


$$
J(s)=\int_{0}^{s} \rho\left(s^{2}-\rho^{2}\right)^{-1 / 2} I(\rho) d \rho .
$$

Substituting for $I(\rho)$ from (1), and assuming that the order of integration can be interchanged, we have

$$
\begin{aligned}
J(s) & =\int_{0}^{s} \rho\left(s^{2}-\rho^{2}\right)^{-1 / 2} \int_{0}^{\infty} f(x) J_{0}(\rho x) d x d \rho \\
& =\int_{0}^{\infty} f(x) \int_{0}^{s} \rho\left(s^{2}-\rho^{2}\right)^{-1 / 2} J_{0}(\rho x) d \rho d x \\
& =\int_{0}^{\infty} x^{-1} f(x) \sin x s d x .
\end{aligned}
$$

Differentiating with respect to $s$, one obtains

$$
J^{\prime}(s)=\int_{0}^{\infty} f(x) \cos x s d x \equiv C(s)
$$

To obtain $I(\rho)$, we use the inversion formula for Abel's integral equation [9, p. 40]

$$
\begin{aligned}
\rho I(\rho) & =\frac{2}{\pi} \frac{d}{d \rho} \int_{0}^{\rho} s\left(\rho^{2}-s^{2}\right)^{-1 / 2} J(s) d s \\
& =\frac{2}{\pi}\left\{J(0)+\rho \int_{0}^{\rho}\left(\rho^{2}-s^{2}\right)^{-1 / 2} J^{\prime}(s) d s\right\} .
\end{aligned}
$$

Since $J(0)=0$, we have, for $\rho \neq 0$,

$$
I(\rho)=\frac{2}{\pi} \int_{0}^{\rho}\left(\rho^{2}-s^{2}\right)^{-1 / 2} J^{\prime}(s) d s .
$$

Also,

$$
I(0)=\int_{0}^{\infty} f(x) d x=J^{\prime}(0)
$$

3. The Numerical Method. Since we can compute approximate values for $J^{\prime}(s)$ by means of the Fourier integral (4), we can use (5) to compute $I(\rho)$. This requires that the integral in (5) be replaced by a numerical quadrature. Because of the singularity of the integrand, we use the technique of product integration [11]. A special case of this technique is explained below, the generalization is obvious.

Suppose we know $J^{\prime}(s)$ for $s_{i}=i h, i=0,1, \cdots, 2 N$. In each interval [ $\left.s_{2 i}, s_{2 i+2}\right]$, we approximate $J^{\prime}(s)$ by quadratic interpolation on the points $s_{2 i}, s_{2 i+1}$, and $s_{2 i+2}$. Then, for $s_{2 i} \leqq s \leqq s_{2 i+2}$,

$$
\begin{aligned}
J^{\prime}(s) \simeq & J_{2 i}^{\prime}+\frac{s-s_{2 i}}{h}\left\{2 J_{2 i+1}^{\prime}-1.5 J_{2 i}^{\prime}-.5 J_{2 i+2}^{\prime}\right\} \\
& +\frac{\left(s-s_{2 i}\right)^{2}}{2 h^{2}}\left\{J_{2 i}^{\prime}-2 J_{2 i+1}^{\prime}+J_{2 i+2}^{\prime}\right\}
\end{aligned}
$$

where $J_{i}^{\prime}$ denotes $J^{\prime}\left(s_{i}\right)$. Using the notation 


$$
Q_{j}(x, t, i)=\frac{1}{h^{j}} \int_{s_{i}}^{s_{i}+t h}\left(x^{2}-s^{2}\right)^{-1 / 2}\left(s-s_{i}\right)^{j} d s,
$$

we have, for $S_{2 k} \leqq \rho \leqq S_{2 k+2}$,

$$
\begin{array}{r}
I(\rho) \simeq \frac{2}{\pi} \sum_{i=0}^{k-1}\left\{J_{2 i}^{\prime} Q_{0}(\rho, 2,2 i)+\left(2 J_{2 i+1}^{\prime}-1.5 J_{2 i}^{\prime}-.5 J_{2 i+2}^{\prime}\right) Q_{1}(\rho, 2,2 i)\right. \\
\left.+\frac{1}{2}\left(J_{2 i}^{\prime}-2 J_{2 i+1}^{\prime}+J_{2 i+2}^{\prime}\right) Q_{2}(\rho, 2,2 i)\right\} \\
+\frac{2}{\pi}\left\{J_{2 k}^{\prime} Q_{0}(\rho, t, 2 k)+\left(2 J_{2 k+1}^{\prime}-1.5 J_{2 k}^{\prime}-.5 J_{2 k+2}^{\prime}\right) Q_{1}(\rho, t, 2 k)\right. \\
\left.+\frac{1}{2}\left(J_{2 k}^{\prime}-2 J_{2 k+1}^{\prime}+J_{2 k+2}^{\prime}\right) Q_{2}(\rho, t, 2 k)\right\},
\end{array}
$$

where $t=\left(\rho-s_{2 k}\right) / h$.

The explicit expressions for the $Q_{i}$ are

$$
\begin{aligned}
Q_{0}(\rho, t, i) & =I_{1}(\rho, t, i), \\
Q_{1}(\rho, t, i) & =I_{2}(\rho, t, i)-i I_{1}(\rho, t, i), \\
Q_{2}(\rho, t, i) & =I_{3}(\rho, t, i)-2 i I_{2}(\rho, t, i)+i^{2} I_{1}(\rho, t, i), \\
I_{1}(\rho, t, i) & =\arcsin ((i+t) / p)-\arcsin (i / p), \\
I_{2}(\rho, t, i) & =\left(p^{2}-i^{2}\right)^{1 / 2}-\left(p^{2}-(i+t)^{2}\right)^{1 / 2}, \\
I_{3}(\rho, t, i) & =.5\left\{i\left(p^{2}-i^{2}\right)^{1 / 2}-(i+t)\left(p^{2}-(i+t)^{2}\right)^{1 / 2}+p^{2} I_{1}(p, t, i)\right\},
\end{aligned}
$$

where $p=\rho / h$.

In practice, most of the computational effort will have to be expended in computing the Fourier integrals $J^{\prime}\left(s_{i}\right)$, for $i=0,1, \cdots, 2 N$. The computation of $I(\rho)$ by Eq. (7) requires much less work. The process is therefore most efficient if the values of $I(\rho)$ are required for a range of $\rho$.

The integral (5) can be transformed into a simpler expression. For example, putting $s=\rho \sin \theta$, we get

$$
I(\rho)=\frac{2}{\pi} \int_{0}^{\pi / 2} J^{\prime}(\rho \sin \theta) d \theta,
$$

which can be handled by standard techniques. Similarly, by a suitable change of variables, the integrand can be written in the form $s^{-1 / 2} g(\rho, s)$ which has been treated in the literature. However, such an approach appears inefficient, since one needs to compute a new set of $J^{\prime}\left(s_{i}\right)$ for each $\rho$, while, with the method outlined in this section, $I(\rho)$ may be computed for a range of $\rho$ with only one set of $J^{\prime}\left(s_{i}\right)$. However, if $I(\rho)$ is needed for only one value of $\rho$, then some simplification can be achieved with the suggested transformation.

The technique outlined in this section is a particular instance of the well-known product integration technique. A more thorough discussion, including the computation of error estimates, may be found in the literature (e.g. Young [11]).

4. A Numerical Example. In Table 1, we summarize the results for

$$
I(\rho)=\int_{0}^{\infty} x\left(x^{2}+1\right)^{-3 / 2} J_{0}(\rho x) d x=e^{-\rho} .
$$


The Fourier integrals were computed by means of an adaptive quadrature method [12] with an estimated accuracy of $10^{-4}$ for $s_{i}=0(.125) 1.0$.

TABLE 1. Results for Numerical Example

\begin{tabular}{ccc}
\hline$\rho$ & computed $I(\rho)$ & $\operatorname{exact} I(\rho)$ \\
\hline \hline .2 & .81868 & .81873 \\
.4 & .67034 & .67032 \\
.6 & .54884 & .54881 \\
.8 & .44934 & .44933 \\
1.0 & .36787 & .36788 \\
\hline
\end{tabular}

5. A Generalization. The above process can be generalized to the evaluation of

$$
I_{\nu}(\rho)=\int_{0}^{\infty} f(x) J_{\nu}(\rho x) d x, \quad \nu=1,2, \cdots .
$$

Using Sonine's first integral [10, p. 373]

$$
J_{\nu+\xi+1}(z)=\frac{z^{\xi+1}}{2^{\xi} \Gamma(\xi+1)} \int_{0}^{\pi / 2} J_{\nu}(z \sin \theta) \sin ^{\nu+1} \theta \cos ^{2 \xi+1} \theta d \theta,
$$

and setting $z=x \xi, r=x \sin \theta, \xi=-\frac{1}{2}$,

$$
\begin{aligned}
\int_{0}^{x}\left(x^{2}-r^{2}\right)^{-1 / 2} r^{\nu+1} J_{\nu}(\xi r) d r & =x^{\nu+1} \frac{(\pi / 2)^{1 / 2}}{(\xi x)^{1 / 2}} J_{\nu+1 / 2}(\xi x) \\
& =x^{\nu+1} j_{\nu}(\xi x),
\end{aligned}
$$

where $j_{v}$ denotes the half-order Bessel function. Since the $j_{v}$ can be expressed in terms of sine and cosine, the process leading to Eqs. (4) and (5) can be repeated with obvious modifications if $\nu$ is an integer.

Department of Mathematics

University of California, Davis

Davis, California 95616

1. S. M. Chase \& L. D. Fosdick, "An algorithm for Filon quadrature," Comm. ACM, v. 12, 1969 , pp. $453-457$.

2. W. W. CLENDENIN, "A method for numerical calculation of Fourier integrals," Numer. Math., v. 8, 1966, pp. 422-436. MR 34 \#982.

3. P. J. Davis \& P. RabiNowITZ, Numerical Integration, Blaisdell, Waltham, Mass., 1967. MR 35 \#2482.

4. A. ERDÉLyI, ET AL., Tables of Integral Transforms. Vol. I, McGraw-Hill, New York, 1954. MR 15, \#868.

5. L. N. G. Filon, “On a quadrature formula for trigonometric integrals," Proc. Roy. Soc. Edinburgh, v. 49,1928 , pp. 38-47.

6. I. M. Longman, "Tables for the rapid and accurate numerical evaluation of certain infinite integrals involving Bessel functions," $M T A C$, v. 11, 1957, pp. 166-180. MR 19, 985.

7. J. N. LYNESS, "The calculation of Fourier coefficients by the Möbius inversion of the Poisson summation formula. I. Functions whose early derivatives are continuous," Math. Comp., v. 24, 1970, pp. 101-135. MR 41 \#4858. 
8. I. N. SNEddon, "Boundary value problems in thermoelasticity," in Boundary Problems in Differential Equations, R. E. Langer (Editor), Univ. of Wisconsin Press, Madison, Wis., 1960, pp. 231-241. MR 32 \#3268.

9. I. N. SNEDDon, Mixed Boundary Value Problems in Potential Theory, North-Holland, Amsterdam; Interscience, New York, 1966. MR 35 \#6853.

10. G. N. Watson, A Treatise on the Theory of Bessel Functions, 2nd ed., Cambridge Univ. Press, Cambridge; Macmillan, New York, 1944. MR 6, 64.

11. A. Young, "Approximate product-integration," Proc. Roy. Soc. London Ser. A, v. 224, 1954, pp. 552-561. MR 16, 179.

12. An adaptive quadrature method based on Filon's method. A FORTRAN program of this algorithm is available from the author. 